\title{
INTRA-ABDOMINAL UMBILICAL VEIN VARIX - A CASE REPORT
}

\author{
Sachin Agrawal ${ }^{1}$, Shraddha Singhania ${ }^{2}$, Pooja Singhania ${ }^{3}$, Kumar Vaibhav $^{4}$ \\ ${ }^{1}$ Consultant Radiologist, Department of Diagnostic Imaging, National University Hospital, Singapore \\ ${ }^{2}$ Assistant Lecturer, Department of Radio- Diagnosis, Jawaharlal Nehru Medical College, Wardha, MAH, India \\ ${ }^{3}$ Consultant Radiologist, Diwan Imaging Centre, Ghaziabad, UP, India \\ ${ }^{4}$ Assistant Professor, Department of Radio- Diagnosis, Jawaharlal Nehru Medical College, Wardha, MAH, India
}

\begin{abstract}
The incidence of the fetal intra-abdominal umbilical vein varix condition is very rare and has been associated with fetal hydrops, IUGR and still birth A 26-year-old primigravida was referred for routine antenatal scan. The scan at 30 weeks showed an intra-abdominal ovoid structure superior to the fetal bladder. Color flow Doppler revealed venous flow in continuity with the umbilical vein. A diagnosis of umbilical varix was made. The venous flow was present throughout the lesion, suggesting the absence of thrombi. There was no evidence of fetal hydrops. Subsequent scans at regular intervals showed no increase in size of the umbilical varix. The patient had an uneventful elective cesarean section at 39 weeks. Postnatal assessment and a follow-up neonatal cardiac echo scan were normal. Our case supports the new emerging evidence that pregnancy outcome in cases of isolated fetal umbilical vein varix is generally good. Caution must be exercised against unnecessary early induction and costly preterm births
\end{abstract}

KEYWORDS - Fetal umbilical vein varix, Ultrasonography, Pregnancy outcome

\section{INTRODUCTION}

Fetal intra-abdominal umbilical vein (FIUV) varix is a focal dilatation of the umbilical venous diameter at the level of cord insertion. The diameter of the umbilical vein normally increases linearly with gestational age. However, although FIUV is rare and the cause is currently unknown, the condition can be diagnosed by antenatal ultrasound (1)

A fetal intra-abdominal umbilical vein varix has been described differently by different studies, few studies described it as an index portion of the umbilical vein that is at least 50\% wider than the non dilated portion or dilatation of $9 \mathrm{~mm}$ or greater (2) whereas others have defined it as a measurement that is more than 2 SDs above the mean for gestational age.(3) Ultrasound FIUV varix findings indicate intra-abdominal dilation, which is usually an extra hepatic portion of the umbilical vein. The weakest part of the umbilical circulation is the extra hepatic intra-abdominal portion of the umbilical circulation and therefore any condition that can increase the umbilical venous pressure could potentially induce umbilical vein dilation (4)

The first description of a fetal intraabdominal umbilical vein varix was of several cases with grave fetal outcomes (5) and most of the initial studies were case reports with mortality rates of up to $43 \%$.(6,7)

In recent reports, the neonatal prognosis was found to be substantially better than that reported in older studies.(6) Our case supports the new emerging evidence that pregnancy outcome in cases of isolated fetal umbilical vein varix is generally good. Caution must be exercised against 
unnecessary early induction and costly preterm births.

\section{CASE PRESENTATION}

A 26-year-old woman primigravida was referred to our department at 30 weeks of gestation for routine antenatal scan. A detailed fetal scan showed an intra abdominal cystic lesion located at the level of the umbilicus running in an anteroposterior and caudocephalic direction, slightly on the left of the midline (Figure 1). Sagittal and oblique views revealed that this lesion was in continuity with the umbilical vein. The color Doppler imaging (CDI) indicated blood flow (Figure 2) and Doppler examination confirmed the presence of venous flow within the lesion (Figure 3). A diagnosis of dilatation of the extra hepatic portion of the umbilical vein was made. This was an isolated finding without any associated fetal abnormalities. In CDI, venous flow was present throughout the lesion thus suggesting the absence of thrombi in the vascular lumen. The follow-up examinations at regular intervals did not reveal any change in the size of the umbilical vein dilatation. There were no signs of cardiomegaly or hydrops. A healthy female infant was born at 39 weeks of gestation by elective Cesarean section, and discharged from the hospital after a few days of uneventful recovery of the mother.

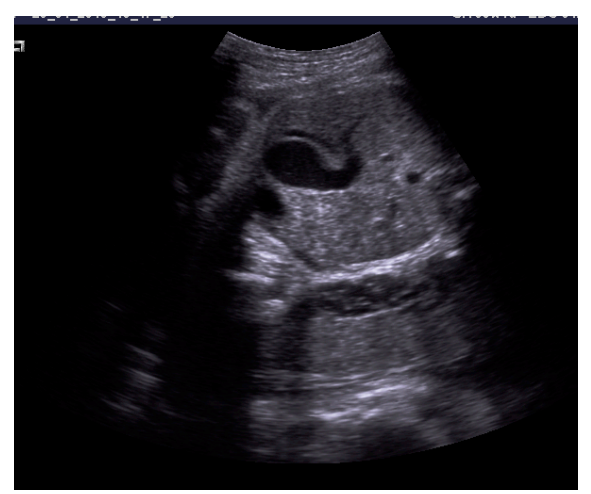

Figure 1: Grey scale USG showing well defined cystic area adjacent to the bladder

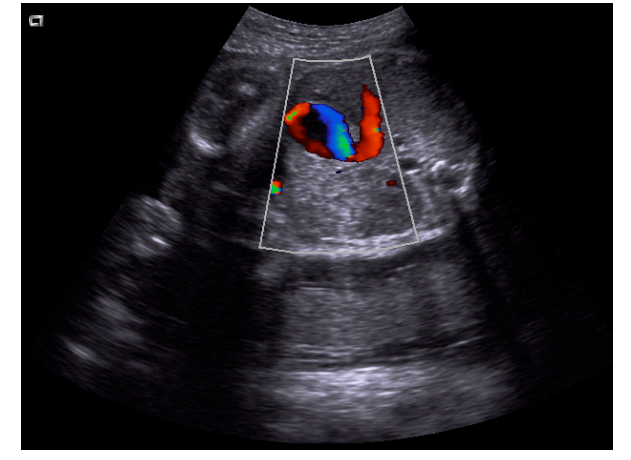

Figure 2: Colour Doppler imaging showed colour flow within the lesion

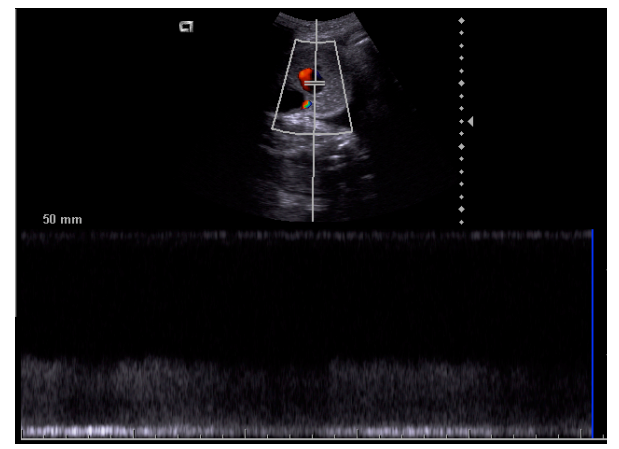

Figure 3: Doppler examination showing venous flow within the lesion

\section{DISCUSSION}

In the embryo, the right and left umbilical veins run on each side of the liver and carry welloxygenated blood from the placenta to the sinus venosus. As the liver develops, the umbilical veins lose their connection with the heart and empty into the liver. The right umbilical vein disappears at the end of the embryonic period, leaving the left umbilical vein as the only vessel carrying welloxygenated blood from the placenta to the embryo and fetus. (7)

A fetal intra-abdominal umbilical vein varix is considered an uncommon prenatal sonographic finding. The frequency of diagnosis has increased substantially over the last 5 years, probably because of increasing awareness of the diagnosis and improved ultrasound technology.

Earlier studies described neonatal mortality rates as high as $43 \%(8,3)$, which caused 
considerable anxiety for patients and medical personnel and were the basis for recommendations for early delivery at 34 weeks. (3) Recent studies, however, have been more reassuring. $(9,10)$

Weissmann-Brenner et al. (10) recently summarized 109 cases of FIUV varices from several different papers and found that the prognosis of 'isolated' FIUV varix or those with associated anomalies were both fairly good. Our case of antenatally diagnosed umbilical vein varix has normal outcome.

\section{CONCLUSION}

Our case supports the new emerging evidence that pregnancy outcome in cases of isolated fetal umbilical vein varix is generally good. Caution must be exercised against unnecessary early induction and costly preterm births. However close monitoring should be done.

\section{REFERENCES}

1. Mantas N, Sifakis S, Koukoura O, Avgoustinakis E, Koumantakis E. Intraabdominal umbilical vein dilatation and term delivery: a case report and review of the literature. Fetal Diagn Ther. 2007;22:431-434.

2. Ipek A, Kurt A, Tosun O, et al. Prenatal diagnosis of fetal
3. Mahony BS, McGahan JP, Nyberg DA, Reisner DP. Varix of the fetal intra-abdominal umbilical vein: comparison with normal. J Ultrasound Med1992; 11:73-76.

4. Nyberg D. Varix of the umbilical vein. In: Nyberg DA, McGahan JP, Pretorius DH, Pilu G, editors.Diagnostic imaging of fetal anomalies. Philadelphia: Lippincott Williams \& Wilkins; 2003. pp. 114-115.

5. Fuster JS, Benasco C, Saad I. Giant dilatation of the umbilical vein. J Clin Ultrasound 1985; 13:363-365.

6. Fung TY, Leung TN, Leung TY, Lau TK. Fetal intraabdominal umbilical vein varix: what is the clinical significance? Ultrasound Obstet Gynecol2005; 25:149-154.

7. Valsky D, Rosenak D, Hochner-Celnikier S, Porat S, Yagel S. Adverse outcome of isolated fetal intra-abdominal umbilical vein varix despite close monitoring. Prenat Diagn 2004; 24:451-454

8. Moore KL, Persaud TVN. The Developing Human. Clinically Oriented Embryology. Philadelphia: Saunders, 1998: $350-5$

9. Weissmann-Brenner A, Simchen MJ, Moran O, Kassif E, Achiron $R$, Zalel $Y$. Isolated fetal umbilical vein varix: prenatal sonographic diagnosis and suggested management. Prenat Diagn 2009; 29:229-233.

10. Byers BD, Goharkhay N, Mateus J, Ward KK, Munn MB, Wen TS. Pregnancy outcome after ultrasound diagnosis of fetal intra-abdominal umbilical vein varix. Ultrasound Obstet Gynecol 2009; 33:282-286

How to cite this article - Agrawal S, Singhania S, Singhania P et. al. Intra-Abdominal Umblical Vein Varix - A Case Report, IJRSMS, 2015;01(1): 12 - 14 